\title{
Multistep and multiscale electron transfer and localization dynamics at a model electrolyte/metal interface
}

\author{
Sarah B. King, ${ }^{1, \text { a) }}$ Katharina Broch, ${ }^{1, \text { b) }}$ Angelika Demling, ${ }^{1}$ and Julia Stähler ${ }^{1, c)}$ \\ Department of Physical Chemistry, Fritz Haber Institute of the Max Planck Society, Faradayweg 4-6, 14195 Berlin, \\ Germany
}

(Dated: 4 October 2018)

The lifetime, coupling, and localization dynamics of electronic states in molecular films near metal electrodes fundamentally determine their propensity to act as precursors or reactants in chemical reactions, crucial for a detailed understanding of charge transport and degradation mechanisms in batteries. In the current study, we investigate the formation dynamics of small polarons and their role as intermediate electronic states in thin films of dimethyl sulfoxide (DMSO) on $\mathrm{Cu}(111)$ using time- and angle-resolved two-photon photoemission spectroscopy. Upon photoexcitation, a delocalized DMSO electronic state two monolayers from the $\mathrm{Cu}$ surface is initially populated, becoming a small polaron on a 200 fs timescale, consistent with localization due to vibrational dynamics of the DMSO film. The small polaron is a precursor state for an extremely long-lived and weakly-coupled multilayer electronic state, with a lifetime of several seconds, thirteen orders of magnitude longer than the small polaron. Although the small polaron in DMSO has a lifetime of 140 fs, its role as a precursor state for long-lived electronic states could make it an important intermediate in multistep battery reactivity.

\section{INTRODUCTION}

The localization and lifetime of electronic states influences charge transport processes and chemical reactivity in a variety of ways, including molecular charge re-distribution dynamics, dynamics of electron and hole transport in semiconductors, surface state reactions, or catalysis. ${ }^{1-3}$ As more research has focused on organic semiconductors and novel battery technologies, scientific interest has increased into the mechanism and timescale of the formation of localized electronic states in molecular environments from delocalized electronic states, and how this modifies interface functionality and chemical reactivity. ${ }^{4-13}$ In electrochemical cells, electron transfer from an electrode into an electrolyte depends upon effective wavefunction overlap between delocalized Bloch states of the metal electrodes and delocalized electrolyte electronic states. ${ }^{14}$ Chemical reactivity of electronic states in the electrolyte, in contrast, requires electronic states with long-lifetimes and thus decoupling of electrolyte and metal electronic states. Therefore transient electrolyte electronic states at the interface, with dynamic localization, can be important intermediates for the functionality of electrochemical interfaces.

Dimethyl sulfoxide (DMSO), see Figure 1(a), is a commonly used battery electrolyte in development efforts of lithium-oxygen batteries. ${ }^{15-18}$ DMSO is a polar aprotic molecule with one of the highest dielectric constants of common solvents, $\epsilon=47$, and a large $4.3 \mathrm{D}$ dipole

\footnotetext{
a) Present address: University of Chicago, Department of Chemistry and James Franck Institute, 929 E 57th Street, Chicago, IL 60637, USA; Electronic mail: sbking@uchicago.edu

b) Present address: Universität Tübingen, Institut für Angewandte Physik, Auf der Morgenstelle 10, 72076 Tübingen, Germany

c) Electronic mail: staehler@fhi-berlin.mpg.de
}

moment. ${ }^{19}$ The dipole moment points slightly off angle of the sulfur-oxygen bond. ${ }^{20}$ The ordering of DMSO at electrolyte surfaces varies depending upon the electrode potential, solute identity, and charge, ${ }^{18,21,22}$ stoking interest in how DMSO dynamically interacts with charged species and screens charge. In order to decouple the influence of solutes and co-adsorbates, studies have focused on the interaction and dynamics of DMSO with metal surfaces and excess electrons using surface science techniques. $^{23-26}$ Structural studies of DMSO on noble metal surfaces show that DMSO orders to the metal surface, binding in either a mono- or bi-dentate manner through the sulfur or the sulfur and oxygen lone pairs, ${ }^{23-25}$ while maintaining long-range order between chains of DMSO molecules. After electron injection from a $\operatorname{Ag}(111)$ surface into one and two layers of DMSO, image potential states (IPS) of Ag modified by the layers of DMSO are initially formed. These states are delocalized parallel to the metal surface with nearly-free electron effective masses before collapsing into localized electronic states within 800 fs via frustrated rotation of the DMSO molecules. ${ }^{26}$ This study focused on the dynamics in ultrathin DMSO layers, and the observed ultrafast dynamics were highly sensitive to the DMSO coverage.

The lifetime of an electronic state is important in determining the probability of charge transfer to other electronic states, and whether it is chemically reactive. Previous ultrafast surface science investigations have shown that the dynamics of an electronic state in a molecular film can differ based on the molecular properties of the film, the electronic state localization, influence of the metal substrate, and molecular film thickness. ${ }^{8,10,27-35}$ The contrasting dynamics of electron localization in nonpolar n-heptane versus polar water and ammonia highlight the influence of the molecular properties of the film. In ultra-thin non-polar n-heptane layers on a $\mathrm{Ag}(111)$ surface, electron injection leads to the formation of small 


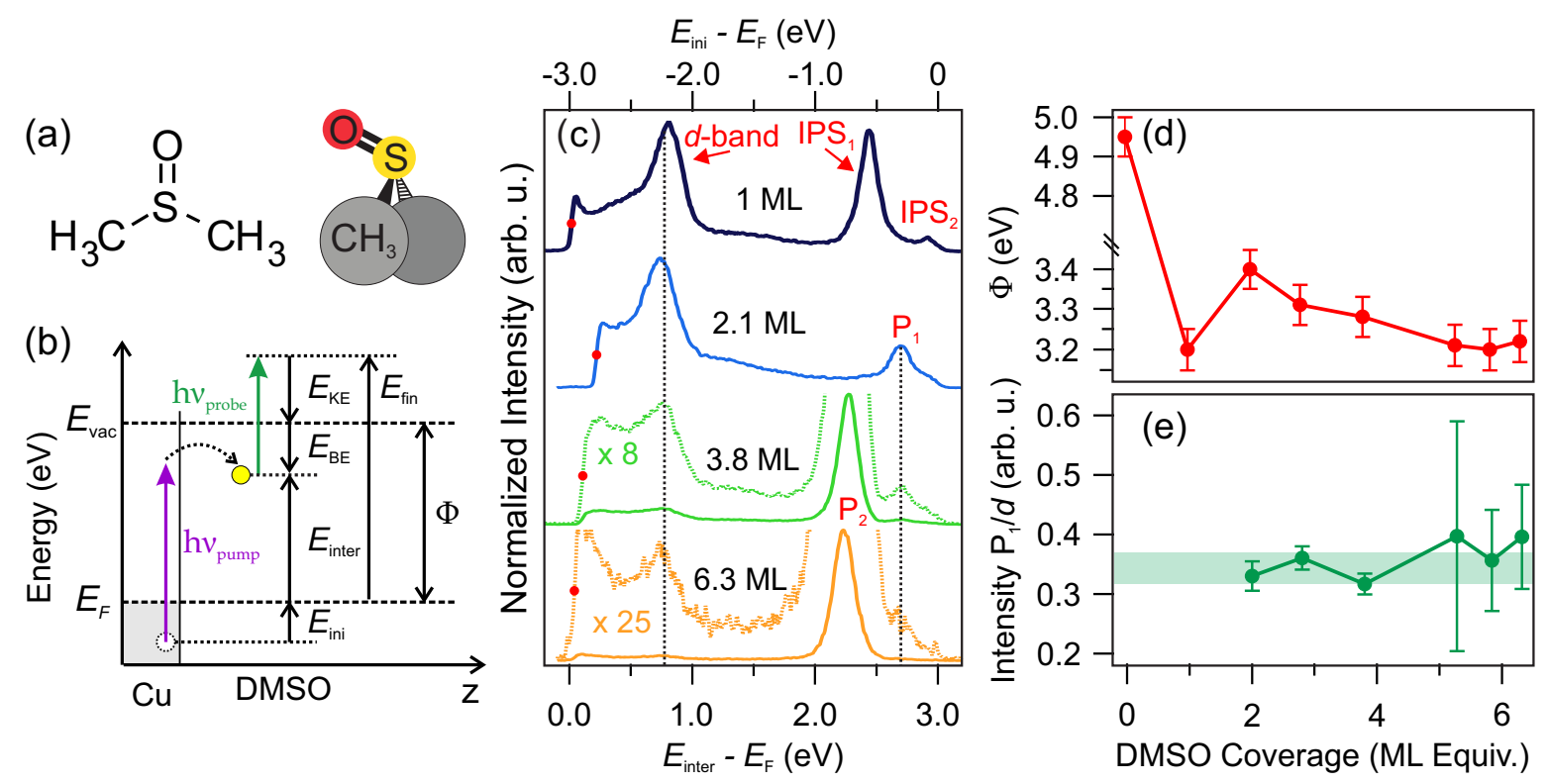

FIG. 1. (a) Kekulé structure and 3D representation of dimethyl sulfoxide. (b) The experimental scheme for TR-2PPE including the relevant energy definitions. (c) Static one-color two-photon photoemission spectra of 1 to $6.3 \mathrm{ML}$ of $\mathrm{DMSO}$ on $\mathrm{Cu}(111)$. (d) The work function of DMSO/Cu(111) versus DMSO coverage in ML. (e) The intensity ratio of $P_{1}$ to the $d$-bands for DMSO $\geq 2 \mathrm{ML}$, with the error-weighted-average of 0.33 shown by the green rectangle.

polarons that are self-trapped via the in-phase methylene rocking of n-heptane on an approximately $360 \mathrm{fs}$ timescale. ${ }^{27} \mathrm{~A}$ small polaron is a quasi-particle consisting of an excess electron dressed by the polarization it induces in the material. This material polarization increases the electron binding energy and results in charge localization to dimensions of a molecule or crystalline unit cell. ${ }^{4,36,37}$ In frozen water and ammonia, electrons form solvated electrons in pre-existing electron traps on ultrafast timescales. ${ }^{28,31,34}$ Solvated electrons are electrons cooperatively supported by the dipole moments of the solvent and are typically described in a cavity or pseudo-cavity model. ${ }^{38,39}$

For many electronic states near metal interfaces, recombination with the metal substrate is the primary decay mechanism that determines the electronic state lifetime. The coupling of electronic states to metal substrates can be strong and is dependent on the localization of the electronic state, the momentum-resolved band structure of the metal, and the specific molecular geometry at the interface. ${ }^{23-25,28}$ For example, delocalized electronic states of amorphous solid water have electron transfer rates to the metal substrate that are dependent on the metal electronic structure. Localized electronic states often do not have the same substrate dependence, due to reduced substrate coupling; rather the lifetime is determined by the screening of the molecular film. ${ }^{28}$ Thick molecular films can improve stabilization mechanisms such as electron solvation and increase the lifetime of an electronic state through enhanced screening. In both frozen ammonia and ionic liquids, for instance, the lifetime of the localized solvated electron increases as a function of coverage as the electrons are separated from the metal by more and more dielectric material, which reduces the electron transfer rate. ${ }^{31,40,41}$ In molecular films with strong screening this can lead to states with lifetimes on the order of seconds to minutes, as observed in crystalline ice, crystalline ammonia, and amorphous solid water. ${ }^{31,42-45}$ The extremely long lifetimes of these normally unoccupied electronic states is sufficient for chemical reactivity both within molecular layers as well as with molecules exposed from the gas-phase. ${ }^{43-45}$

In DMSO in electrochemical cells, transient interactions of the first few layers of solvent molecules due to changing electrode potentials can modify interface electronic states, their lifetimes, and coupling with reactants on ultrafast timescales, strongly determining the cell's functionality. ${ }^{15,18}$ Therefore, for a complete description of the role of DMSO in electrochemical and battery systems it is important to understand the localization, coupling, and lifetime of DMSO electronic states near metal electrodes, and how DMSO electronic states near the interface could be precursors for long-lived and chemically reactive electronic states. In this paper, we investigate dynamic electron localization in 2-6 monolayer DMSO films on the $\mathrm{Cu}(111)$ surface using time- and angleresolved two-photon photoemission (TR-AR-2PPE). By using this surface-science technique we are able to investigate the ultrafast dynamics of excess electrons in pure DMSO, without the influence of solute or counter ions, as a function of DMSO coverage and distance from a metal substrate. We observe the dynamic localization and formation of a small polaron on a 200 fs timescale that is located approximately 2 ML from the metal sur- 
face and is strongly coupled to the substrate. Furthermore, we find the small polaron is a precursor state for an extremely long-lived, localized, and potentially-reactive electronic state at the DMSO/vacuum interface formed at coverages $>2 \mathrm{ML}$ of DMSO. This work shows how an electronic state at an electrolyte/metal interface could be an important intermediate for electrochemical reactivity even if it has a femtosecond lifetime.

\section{EXPERIMENTAL METHODS}

The $\mathrm{Cu}(111)$ crystal was prepared by repeated cycles of sputtering at $0.7 \mathrm{kV}$ with $1.5 \times 10^{-6} \mathrm{mbar} \mathrm{Ar}^{+}$ions for 10 minutes followed by annealing at $800 \mathrm{~K}$ for 45 minutes. The cleanliness of the surface was verified by LEED, work function measurements, and the intensity and width of the $\mathrm{Cu}(111)$ surface state in photoemission spectra of the clean surface. The $\geq 99.9 \%$ anhydrous dimethyl sulfoxide was purchased from Sigma Aldrich, transferred to a glass sample tube on our gas manifold under Argon atmosphere and purified by repeated freeze-pump-thaw cycles. The cleanliness of the DMSO was verified by residual gas analysis. The DMSO was dosed onto the $\mathrm{Cu}(111)$ surface using a pinhole doser and the coverage was verified with temperature programmed desorption (TPD). Details of the dosing procedures and coverage measurements are provided in the supporting material.

One of two laser systems was used, either a Coherent Vitara oscillator and RegA regenerative amplifier which produces $40 \mathrm{fs}$ pulses at a central wavelength of $800 \mathrm{~nm}$ coupled to an optical parametric amplifier (OPA), or a Light Conversion Pharos with a central wavelength of $1030 \mathrm{~nm}$ coupled to two OPAs which produce $30 \mathrm{fs}$ pulses at variable wavelengths covering $940 \mathrm{~nm}$ to $250 \mathrm{~nm}$. Both lasers have repetition rates of $200 \mathrm{kHz}$. Two different photon energies were used, $3.1 \mathrm{eV}$ and $2.2 \mathrm{eV}$. Both photon energies were re-compressed using prism pairs resulting in a cross-correlation of $58 \pm 8 \mathrm{fs}$, fit with a $\operatorname{sech}^{2}(t / \tau)$ function, and measured in the UHV chamber on the clean $\mathrm{Cu}$ crystal. Pulse energies between 0.6-1.3 nJ/pulse and $0.5-1.4 \mathrm{~nJ} /$ pulse at a $200 \mathrm{kHz}$ repetition rate were used for the $3.1 \mathrm{eV}$ and $2.2 \mathrm{eV}$ photon energies, respectively.

TR-AR-2PPE has been described in great detail elsewhere, ${ }^{46}$ but a brief discussion follows. An electron is excited with a femtosecond laser pump pulse from an occupied electronic state below the Fermi level $\left(E_{\mathrm{F}}\right)$ to an unoccupied electronic state, as shown in Figure 1(b). A second pulse, delayed in time relative to the first, then probes the resulting non-equilibrium electron distribution using photoemission. The photoelectrons are detected using a hemispherical electron analyzer with an acceptance angle of \pm 13 degrees from SPECS Surface Nano Analysis GmbH. The angle of photoemission is related to the parallel momentum of the resulting photoelectrons which is conserved upon photoemission, as described in the supporting information. The total energy resolution of the hemispherical ana- lyzer and laser system is $50 \mathrm{meV}$. As shown schematically in Figure 1(b), the electron kinetic energy $\left(E_{\mathrm{KE}}\right)$ is related to the final $\left(E_{\mathrm{fin}}\right)$, intermediate $\left(E_{\text {inter }}\right)$, and initial state energy $\left(E_{\text {ini }}\right)$ of the electrons relative to the the Fermi level of the $\mathrm{Cu}$ substrate, by the following: $E_{\text {fin }}-E_{\mathrm{F}}=E_{\mathrm{KE}}+\Phi, E_{\text {inter }}-E_{\mathrm{F}}=E_{\mathrm{KE}}+\Phi-h \nu_{2}$, and $E_{\mathrm{ini}}-E_{\mathrm{F}}=E_{\mathrm{KE}}+\Phi-h \nu_{2}-h \nu_{1}$, where $\Phi$ is the work function of the sample. The intermediate energy is related to the vertical binding energy of an electron by the work function, $E_{\mathrm{BE}}=\Phi-\left(E_{\text {inter }}-E_{\mathrm{F}}\right)$.

Pump-wait-probe measurements, as described in detail in references ${ }^{45,47}$ and the supporting information, were used to determine the lifetimes of electronic states that survive longer than the inverse repetition rate of the laser system, $5 \mu \mathrm{s}$. The Xe titration measurements were performed by monitoring the photoemission signal as a function of real-time during exposure to Xe gas. The Xe gas was leaked into the vacuum chamber at pressures between $4 \mathrm{E}-9$ and $6 \mathrm{E}-9$ mbar. The coverage of Xe was calibrated from comparison of integrated Xe TPD on a clean $\mathrm{Cu}$ surface where Xe monolayers and multilayers can be clearly distinguished due to different desorption temperatures. ${ }^{48}$

\section{RESULTS AND DISCUSSION}

\section{A. Unoccupied electronic states of DMSO}

First we address the normally unoccupied DMSO electronic states as a function of DMSO coverage. Static single-color two-photon photoemission spectra of 1-6.3 DMSO monolayers on the $\mathrm{Cu}(111)$ surface with $3.1 \mathrm{eV}$ photon energy are shown in Figure 1(c) with the relevant initial and intermediate state energy axes. Three different peaks are observed in the $1 \mathrm{ML}$ static spectrum. The peak at $-2.17 \pm 0.05 \mathrm{eV}$ (top axis) with respect to $E_{\mathrm{F}}$ is due to $2 \mathrm{PPE}$ with $3.1 \mathrm{eV}$ from the occupied $d$-bands of the $\mathrm{Cu}$ substrate, labeled $d$-band. ${ }^{49}$ The two unoccupied intermediate states, labeled IPS 1 and $\mathrm{IPS}_{2}$, are bound with respect to $E_{\text {vac }}$ by $0.65 \pm 0.05$ and $0.17 \pm 0.05 \mathrm{eV}$ and IPS 1 has an effective mass $\left(m^{*}\right)$ of $1.29 \pm 0.02$ times the mass of a free-electron (not shown), consistent with the $n=1$ and $n=2$ image potential states (IPS) of $\mathrm{Cu}$ modified by a layer of DMSO, as observed in previous experiments of a DMSO monolayer on $\mathrm{Ag} .{ }^{26,50}$

The position of the intensity-half-maximum of the secondary edge is marked with a red dot in Figure 1(c); it is equal to the work function of the $\mathrm{DMSO} / \mathrm{Cu}$ system in the final state energy axis (not shown), and varies as a function of DMSO coverage, as shown in Figure 1(d). The significant $1.7 \mathrm{eV}$ reduction in work function from the $4.95 \pm 0.05 \mathrm{eV}$ of clean $\mathrm{Cu}(111)$ is due to the orientation of the DMSO dipole with respect to the $\mathrm{Cu}$ surface and static charge transfer due to DMSO/Cu interaction. Similar to DMSO on Au, DMSO orients and binds to the $\mathrm{Cu}$ surface through the sulfur and oxygen lone pairs with the methyl groups pointing away from the surface in an 
upright configuration. ${ }^{25,51,52}$

The single-color 2PPE spectrum of $2 \mathrm{ML}$ of DMSO has an increased work function of $3.40 \pm 0.05 \mathrm{eV}$, a peak due to the $\mathrm{Cu} d$-bands, and a peak $P_{1}$ at $2.81 \pm 0.05$ $\mathrm{eV}$, with positive dispersion that can be fit with $m^{*}=$ $1.79 \pm 0.07 \mathrm{~m}_{e}$. The positive dispersion of $P_{1}$ indicates that it is a delocalized electronic state. As the coverage is further increased, the work function decreases again, leveling off at approximately $3.22 \pm 0.05 \mathrm{eV}$ as shown in Figure 1(d) while both the energy of $P_{1}$ and the intensity ratio between $P_{1}$ and the $d$-bands, as shown in Figure 1(e), remain constant. At DMSO coverages $>2$ ML a new peak $P_{2}$ appears in the static spectra and increases in intensity with increasing coverage, peaking at $2.34 \pm 0.05 \mathrm{eV}$ and with slight negative dispersion that can be fit with $m^{*}=-8 \pm 4 \mathrm{~m}_{e}$. As discussed in detail in the following section, slight negative dispersion is a spectroscopic signature for localized electronic states with wide energy distributions. In contrast to $P_{1}, P_{2}$ is a localized electronic state. The multilayer films require annealing to $180 \mathrm{~K}$, as discussed in the supporting information, for the clear appearance of $P_{1}$ and $P_{2}$. The increase in the work function of the $\mathrm{DMSO} / \mathrm{Cu}$ system upon the addition of the second ML suggests that the second layer at least partially orders in an opposite manner to the first layer, where the dipole moments of the second layer are anti-parallel to the first. ${ }^{24}$ Similar to other measurements, ${ }^{23-25,52}$ we do not expect ordered layerby-layer growth for higher coverages and the subsequent reduction of the work function with further increasing coverage is likely due to a decrease in the ordering enforced by the $\mathrm{Cu}$ surface as interactions between DMSO molecules become more important further from the $\mathrm{Cu}$ substrate..$^{53}$

\section{B. Ultrafast small polaron formation dynamics}

In the ultrafast dynamics of multilayers of DMSO on $\mathrm{Cu}(111)$ a small polaron is formed on a $200 \mathrm{fs}$ timescale approximately $2 \mathrm{ML}$ from the $\mathrm{Cu}$ substrate, observed by the dynamic stabilization and localization of $P_{1}$ as a function of pump-probe time delay. Figure 2(a) shows the time-resolved 2PPE intensity of 6.3 ML of DMSO on $\mathrm{Cu}(111$ ) (from here referred to as $6 \mathrm{ML}$ ) measured with $3.08 \mathrm{eV}$ pump energy, $h \nu_{1}$, and $2.19 \mathrm{eV}$ probe energy, $h \nu_{2}$. The same time-resolved spectrum for 2.1 ML of DMSO (from here referred to as $2 \mathrm{ML}$ ) is shown in the supporting information.

Spectra at pump-probe delay times up to 350 fs are shown in the colored solid lines in Figure 2(b). Photoemission signal correlated with pump-probe delay time can be seen up to $3.08 \mathrm{eV}$ intermediate state energy, labeled $P_{1}\left(h \nu_{1}+h \nu_{2}\right)$. Naturally, at $t=0$ the peak energy of the time-resolved $P_{1}$ is the same as $P_{1}$ in the static 2PPE spectrum, however large changes in energy and intensity of $P_{1}$ occur as a function of pump-probe timedelay (the shifting in energy can be clearly seen in Figure $2(\mathrm{~b}))$.

The energy of $P_{1}$ with respect to $E_{\mathrm{F}}$ strongly decreases with pump-probe time delay (equivalent to an increase in binding energy of $P_{1}$ with respect to $\left.E_{\mathrm{vac}}\right)$. Figure 3(a) shows the energy of the $P_{1}$ peak maximum around the $k_{\|}=0$ point as a function of pump-probe delay time where the shaded region reflects the uncertainty in the energy of the peak maximum. There is a marked change in shifting of the peak energy at approximately $200 \mathrm{fs}$, which can be captured by fitting the peak energy to a broken line. For the 6 ML peak energy shifting shown in Figure $3(\mathrm{a})$, the change in slope from $-0.84 \pm 0.15 \mathrm{meV} / \mathrm{fs}$ to $-0.11 \pm 0.11 \mathrm{meV} / \mathrm{fs}$ occurs at $230 \pm 40 \mathrm{fs}$ and $2.64 \pm 0.05 \mathrm{eV}$. The two shifting regions in Figure 3(a) are marked by the light and dark gray rectangles and referred to as regions I and II, respectively.

Concurrent with the change in binding energy of $P_{1}$, the dispersion of $P_{1}$ changes with pump-probe time-delay. Dispersion of an electronic state is a measure of the degree of electron (de)localization. ${ }^{54}$ Figure $3(\mathrm{~b})$ shows the Gaussian fit center of $P_{1}$ for the $6 \mathrm{ML}$ data as a function of parallel momentum for selected pump-probe time delays. Representative fits at multiple $k_{\|}$for three different pump-probe time delays are shown in the supplementary information. At $t=40 \mathrm{fs}$, where the intensity of $P_{1}$ is maximized, the dispersion can be fit with $m^{*}$ is $1.85 \pm 0.13 \mathrm{~m}_{e}$. With increasing pump-probe time delay the dispersion of $P_{1}$ flattens, and can be fit with $m^{*}$ is $12 \pm 4 \mathrm{~m}_{e}$ by $160 \mathrm{fs}$, evidence of electron localization. It is important to note that upon electron localization the free-electron approximation no longer holds such that "effective mass" does not have the meaning that it does in band theory of solids, ${ }^{55}$ however we keep using $m^{*}$ in order to have a quantitative description of the dispersion curvature. The dispersion of $P_{1}$ subsequently remains flat, albeit with slightly negative curvature, until 250 fs where $P_{1}$ exhibits marked negative curvature fit with $m^{*}$ of $-4.3 \pm 0.6 \mathrm{~m}_{e}$. The dispersion at $300 \mathrm{fs}$ and $400 \mathrm{fs}$ (not shown) remains the same as at $250 \mathrm{fs}$. The two dispersion regions in Figure 3(b) are marked by the light and dark gray rectangles and match the two shifting regions from Figure 3(a).

Negative dispersion is due to strong electron localization in real space, which leads to a wide intensity distribution in momentum space. With a hemispherical electron analyzer the angle of photoemission is measured and mapped to parallel momentum by equation (1).

$$
\hbar k_{\|}=\sqrt{2 m_{e} E_{\mathrm{KE}}} \sin \alpha
$$

As discussed in detail in reference 54, any data taken at angles other than zero will have influence from the distribution in momentum space, leading to an intensity weighting of the low-energy portion of the photoemission peak at higher angles. This leads to an apparent negative dispersion, which is an experimental artifact in TR-AR2PPE of localized electronic states at low kinetic energies.

We interpret the continuous binding energy increase of $P_{1}$ and simultaneous electron localization observed in 


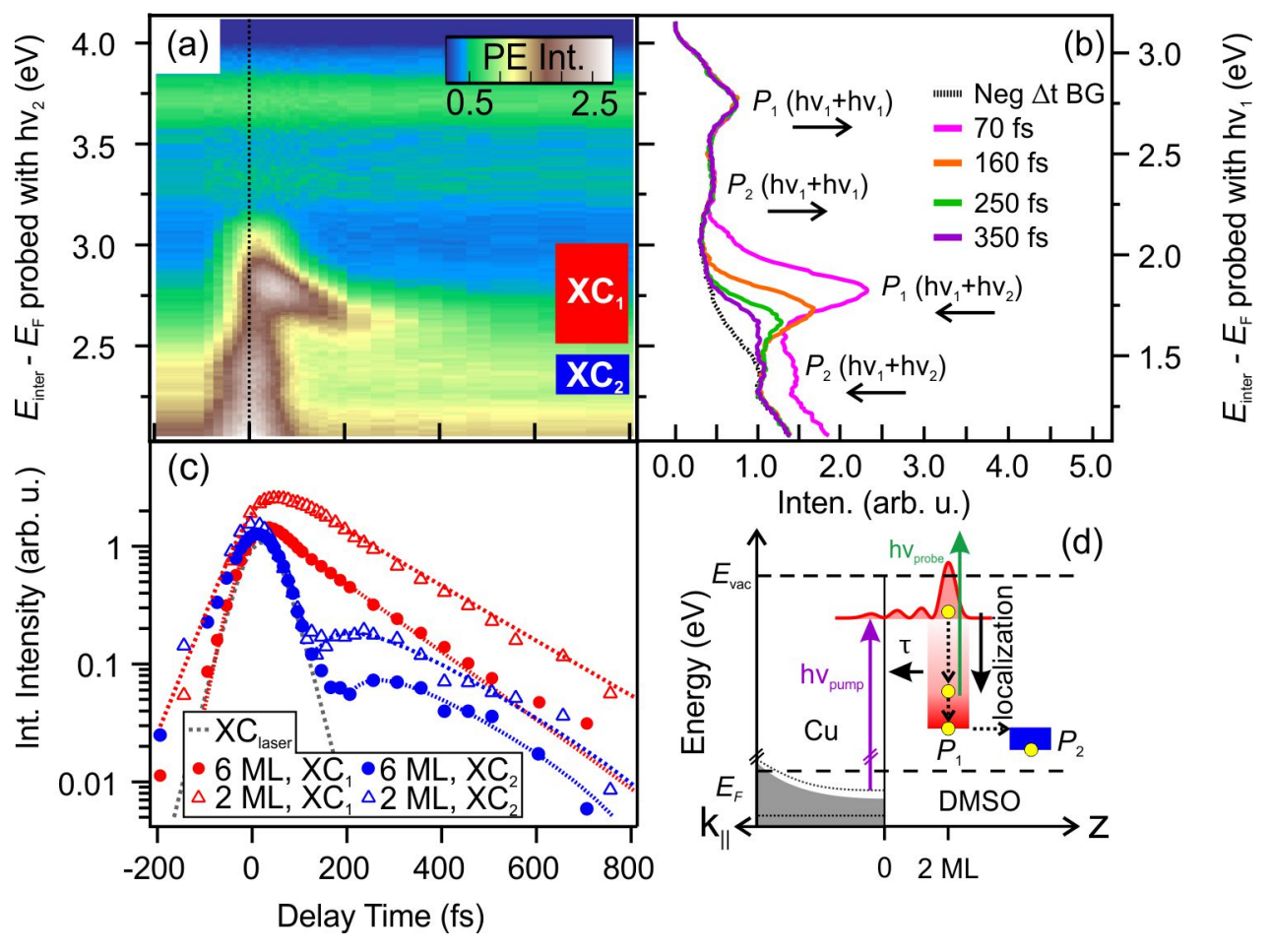

FIG. 2. (a) Time-resolved 2PPE experiment for 6.3 ML of DMSO on $\mathrm{Cu}(111)$ with $h \nu_{1}=3.08 \mathrm{eV}$ and $h \nu_{2}=2.19 \mathrm{eV}$ integrated over $k_{\|}= \pm 0.06 \AA^{-1}$ (raw data) without negative-time background subtraction. The dotted line shows time-zero and the blue and red rectangles are the integration regions for traces in (c). Spectra from (a) at various pump-probe delay times are shown in (b) including: the negative time background between -50 to $-7 \mathrm{ps}$, and positive delays at $70 \mathrm{fs}, 160 \mathrm{fs}, 250 \mathrm{fs}$, and $350 \mathrm{fs}$, with the single-color peaks $P_{1}$ and $P_{2}$, and the correlated peak $P_{1}$. The arrows refer to the relevant intermediate state energy axis depending on the photon energy leading to photoemission. (c) Cross-correlation (XC) traces of the regions indicated in part (a) for 6 and 2 ML coverages. The laser cross-correlation is shown in gray. (d) The Cu(111) occupied electronic band structure versus $k_{\|}$. Schematic of excitation to $P_{1}$, small polaron formation, and formation of $P_{2}$ versus $z$. $\tau$ captures the electron transfer of $P_{1}$ back to the $\mathrm{Cu}$ substrate. Note the $\mathrm{x}$ axis is divided and energy axis is not to scale.

region I as small polaron formation in DMSO on an approximately 200 fs timescale, shown schematically in Figure 2(d). Injection of an electron into DMSO induces a change in the polarization due to molecular vibrations which in turn leads to an increase in the binding energy of the electron, and the formation of a small molecular polaron. This is a dynamic process that occurs on the timescales of the molecular modes linked to polaron formation. ${ }^{56}$ The typical energy gain for the formation of a small polaron due to polarization induced by a change in intramolecular coordinates is on the order of $100-200 \mathrm{meV},{ }^{56}$ consistent with the approximately $150 \mathrm{meV}$ binding energy increase observed in region I of Figure 3(a). The continuous increase in electron binding energy with a rapid increase in localization are consistent with continuous polaron formation through a cross-over region as discussed in the literature. ${ }^{6,9,12}$ Such a continuous localization has been calculated to also occur in liquid water, where the radius of gyration continuously decreased with solvation of the excess electron within 200 fs. ${ }^{57}$ The shifting behavior of $P_{1}$ in region II, we attribute to longer-range screening of the small polaron over larger dimensions, ${ }^{8}$ which further increases the binding energy of the small polaron. In this context, screening refers to molecular reorientation at larger distances from the small polaron that contribute to further localization and that is reflected in continuous binding energy gain. ${ }^{58}$ Similar energy shifting has been observed in electron solvation in amorphous solid water and ammonia. ${ }^{28,34}$

We do not see evidence of simultaneous delocalized and localized electronic states, as suggested in Strader et al. ${ }^{26}$ even in photoemission spectra with high $k_{\|}$, as shown in the supporting information. However the overall similarities of the electronic states in the two studies, both in terms of rough localization dynamics and energetics, suggests that ultrafast localization dynamics are a ubiquitous presence in DMSO electronic states near metal surfaces.

There is debate as to the exact connection between the "polaron formation time" and the period of molecular modes - the timescales discussed range from a quarter to several vibrational or rotational periods. ${ }^{9,10}$ In $n$-heptane small polaron formation was observed due to methylene rocking on a $360 \mathrm{fs}$ timescale. ${ }^{27}$ When the vibrational modes coupled to polaron formation have slow frequencies and result in a large induced polarization, polaron 

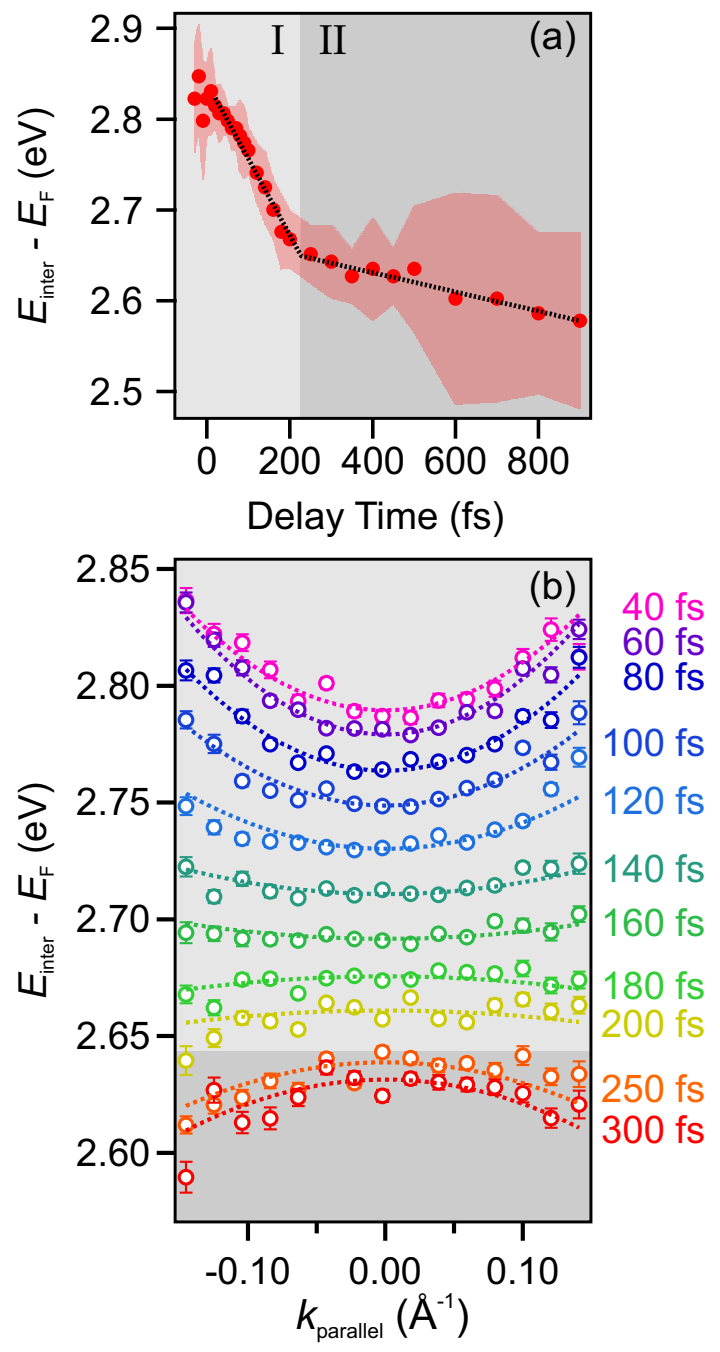

FIG. 3. (a) The peak center of $P_{1}$ at $k_{\|}= \pm 0.06 \AA^{-1}$ versus pump-probe time delay and a fit to a broken line. (b) The peak center of $P_{1}$ versus parallel momentum, $k_{\|}$, fit to a freeelectron dispersion with a varying $m^{*}$ as a function of pumpprobe time delay. Each cut was an average over $0.02 \AA^{-1}$.

formation is energetically favorable with respect to a delocalized electronic state and there is no energy barrier to polaron formation. ${ }^{59}$ As Raman modes reflect changes in polarizability, while infrared modes reflect changes in dipole moment, Raman modes are more pertinent to the material polarization changes required for polaron formation, although Raman and IR frequencies are within a few wavenumbers of each other in DMSO. ${ }^{60}$ Using the librational and vibrational Raman modes of crystalline and liquid DMSO, which are similar regardless of DMSO phase ${ }^{60,61}$ any of the librational modes with periods ranging from 227-340 fs, ${ }^{60}$ or the $\mathrm{S}=\mathrm{O}$ antisymmetric and symmetric bending modes with periods of 99 and $87 \mathrm{fs},{ }^{61}$ could be responsible for polaron formation in DMSO. Although the direct assignment of which librational or vibrational motions are responsible for polaron formation is difficult, it is clear that the timescale of formation is much too fast for the polaron formation mechanism to be due to rotational reorientation of DMSO molecules which would occur on picosecond timescales. ${ }^{62}$ However, the longer-range screening of the small polaron that occurs in region II could be due to such DMSO rotational reorientation.

The time-dependent intensity change for the region of $P_{1}, X C_{1}$, shown in red in Figure 2(c), exhibits a lifetime of $140 \pm 8$ fs for $6 \mathrm{ML}$ and $190 \pm 13$ fs for $2 \mathrm{ML}$. The laser cross-correlation is shown in gray for reference. Excitation to $P_{1}$ occurs with a single photon of $3.1 \mathrm{eV}$ from occupied states of $\mathrm{Cu}(111)$ that lie approximately $0.3 \mathrm{eV}$ below the Fermi level, near the energy of the pristine $\mathrm{Cu}(111)$ surface state. The cross-correlation $X C_{1}$ shows that $P_{1}$ is abruptly formed at time-zero. This indicates that population of $P_{1}$ occurs via a vertical transition from the $\mathrm{Cu}$ initial states into the portion of the $P_{1}$ wavefunction that extends into the orientational bandgap of $\mathrm{Cu}(111)$ at $2.8 \mathrm{eV}$. The excitation mechanism is shown schematically in Figure 2(d), with the dispersive surface state and $s p$-band, the flat $d$-band of pristine $\mathrm{Cu}$, as well as the relevant initial wavefunction of $P_{1}$ prior to the subsequent localization dynamics.

Analogous to other dynamics of unoccupied solvent electronic states, ${ }^{26-28,31}$ electron transfer back to the $\mathrm{Cu}$ substrate is likely the primary decay pathway for $P_{1}$. The short lifetime of $P_{1}$ indicates that it is strongly coupled to the metal substrate. ${ }^{63}$ As an increase in the distance between $P_{1}$ and the $\mathrm{Cu}$ surface would lead to an increase in the $P_{1}$ lifetime, ${ }^{31,40} P_{1}$ must be approximately the same distance from the $\mathrm{Cu}$ surface at both coverages, namely 2 ML.

The location of $P_{1} 2 \mathrm{ML}$ from the $\mathrm{Cu}$ substrate is also consistent with the constant ratio of $P_{1}$ and the $d$ bands with increasing average DMSO coverage in Figure 1(e). Photoemission is surface sensitive due to the limited mean-free-path of electrons. Overlayers of molecules or material at the vacuum interface attenuate photoemission from material deeper into the bulk. The constant photoemission ratio from the $\mathrm{Cu} d$-bands and $P_{1}$ means that their photoemission intensities are attenuated equally with increasing coverage. This would not be the case if $P_{1}$ could occur larger distances from the $\mathrm{Cu}$ surface with increasing coverage, the increased DMSO coverage would attenuate photoemission from the $\mathrm{Cu} d$ bands more than $P_{1}$, thus increasing the $P 1 / d$-band ratio. Therefore the constant ratio supports that the relative distance between $P_{1}$ and the $d$-bands is unchanged with increasing coverage and $P_{1}$ remains $2 \mathrm{ML}$ from the $\mathrm{Cu}$ surface even with increasing coverage.

There are two possibilities for the binding location of $P_{1}$ in the second monolayer depending on the DMSO layer morphology. Either DMSO is growing in closed layers, meaning that $P_{1}$ is bound within the bulk of the second monolayer, or DMSO grows in a layer-plus-island (Stranski-Krastnov) morphology where $P_{1}$ is bound in 2 ML thick valleys on the vacuum interface. Localization of $P_{1}$ underneath DMSO islands, on the contrary, would 


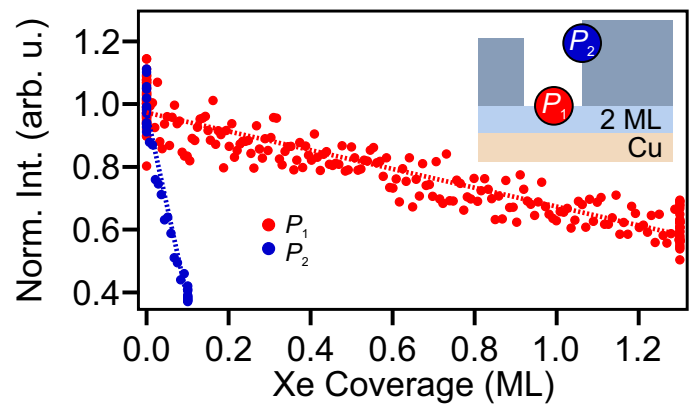

FIG. 4. The normalized relative photoemission intensity of $P_{1}$ with 3.2 ML of DMSO (red) and $P_{2}$ with $4.5 \mathrm{ML}$ of DMSO (blue) to the $\mathrm{Cu} d$-bands as a function of exposure to Xe gas at $44 \mathrm{~K}$.

be in conflict with the constant ratio of $P_{1}$ and the $d$ band intensities from Figure $1(\mathrm{e})$ as the $d$-band signal would be dominated by the $2 \mathrm{ML}$ valleys while $P_{1}$ would be attenuated by increasing DMSO coverage, changing their relative ratio. Whether an electronic state is on the vacuum interface can be determined by exposure of the sample surface to gas phase Xe. Surface bound electronic states can change energy and intensity upon Xe binding due to dielectric squeezing or blocking of electron binding sites by Xe, respectively. As shown in Figure 4, the intensity of $P_{1}$ is reduced by approximately $30 \%$ with 1 ML coverage of Xe, meaning that $P_{1}$ is at least partially exposed to the vacuum interface. Therefore, the DMSO must grow in a layer-plus-island morphology and $P_{1}$ is bound in 2 ML valleys. We are thus observing small polaron formation on a 200 fs timescale in two monolayer valleys between DMSO multilayer islands.

\section{The small polaron as a precursor state for a long-lived surface state}

Now we turn to state $P_{2}$, the normally unoccupied electronic state that occurs for coverages $>2 \mathrm{ML}$. As with $P_{1}$, $P_{2}$ can be bound either at the DMSO/vacuum interface or in the bulk of the multilayer islands and the binding location can be determined by exposure to Xe. Shown in Figure $4, P_{2}$ is strongly quenched by Xe exposure, with a reduction in intensity of over $50 \%$ with only $0.1 \mathrm{ML}$ of Xe. The extremely strong quenching of $P_{2}$ in comparison to $P_{1}$ suggests that Xe preferentially binds to sites on the DMSO surface where $P_{2}$ is located, possibly DMSO surface defects. This preferential adsorption is likely a consequence of the high charge density of the highly localized state $P_{2}$, which attracts Xe due to the polarizability of the rare gas atom.

$P_{2}$ also shows drastically different dynamics compared to $P_{1}$. The presence of $P_{2}$ in the negative time background of the time-resolved experiments indicates that $P_{2}$ has a lifetime that surpasses the inverse repetition rate of the laser system, $5 \mu$ s. The difference in intensity of $P_{2}$ in the static spectra in Figure 1(a) and the negative
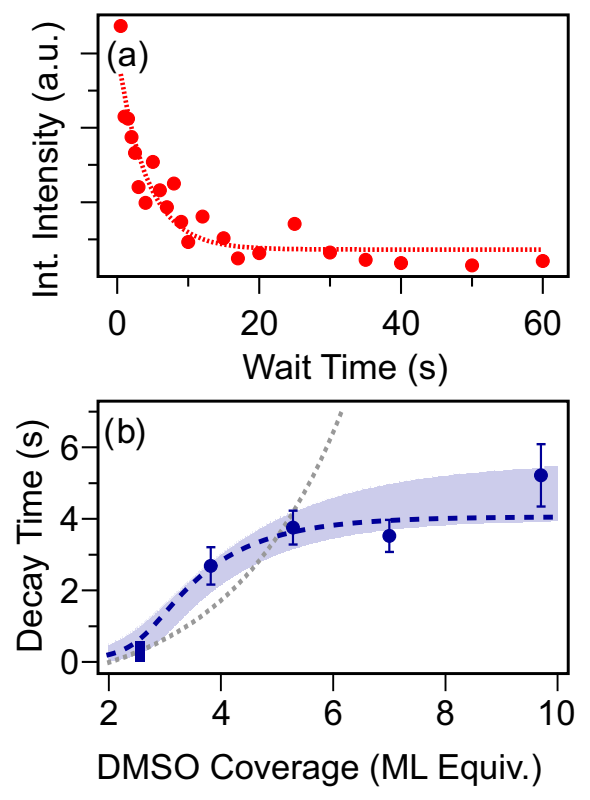

FIG. 5. (a) Representative data of 5.3 ML of DMSO with $\tau=4.1 \pm 0.8 \mathrm{~s}$. (b) The error-weighted-mean values of the decay times versus DMSO coverage including the approximate lifetime of 2.6 ML. The gray line is an exponential function which does not fit the data, while the black line is from a model discussed in the text.

time background in Figure 2(b) is due to a real-time intensity decrease in $P_{2}$ with illumination. This effect was mitigated in the time-resolved data collection. ${ }^{64}$ The long lifetime of $P_{2}$ is confirmed by pump-wait-probe measurements, described in the supporting information, which show that $P_{2}$ has a lifetime of $4.1 \pm 0.8 \mathrm{~s}$ for $5.3 \mathrm{ML}$, as shown in Figure 5(a), thirteen orders of magnitude longer than the lifetime of $P_{1}$. For more information regarding the pump-wait-probe experiments please refer to the supporting online material. The extremely long lifetime of $P_{2}$ means that this state is strongly screened by DMSO and decoupled from the $\mathrm{Cu}$ substrate, similar to surface-bound electrons of amorphous solid water. ${ }^{45}$

The strong screening of $P_{2}$ from the $\mathrm{Cu}$ substrate means that there is negligible wavefunction overlap between $P_{2}$ and electronic states of the $\mathrm{Cu}$ substrate. Therefore, $P_{2}$ cannot be directly populated by an ultrafast laser excitation from occupied electronic states of the $\mathrm{Cu}$ substrate. Instead, an intermediate electronic state is required and $P_{2}$ is likely formed via a higher energy electronic state in DMSO.

The most probable state to form $P_{2}$ is $P_{1}$. This hypothesis is supported by (1) the ultrafast population dynamics, (2) the spectral overlap between $P_{1}$ and $P_{2}$, and (3) the coverage dependence of the $P_{2}$ lifetime that is evidence of wavefunction overlap between $P_{1}$ and $P_{2}$ :

(1) Time-resolved population dynamics: The timedependent intensity change for the region of $P_{2}, X C_{2}$ is shown in Figure 2(c). At early positive delay times, $X C_{2}$ follows the temporal envelope of the laser pulse and 


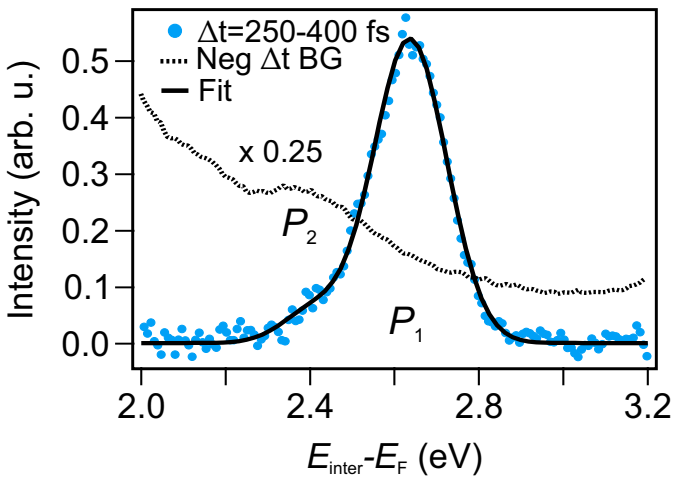

FIG. 6. Average of the background subtracted spectra between 250 and 400 fs (blue dots) with a double Gaussian fit (black line). The negative time background is overlaid for reference (dotted black line).

is due to hot secondary electrons in $\mathrm{Cu}$ that are pumped by $h \nu_{2}=2.19 \mathrm{eV}$ and probed by $h \nu_{1}=3.08 \mathrm{eV}$ (inverse pump-probe scheme) with dynamics that proceed toward negative pump-probe time delays. They have an intermediate energy up to $1.5 \mathrm{eV}$ above the Fermi level (right axis in Figure 2(a)/(b)), far away from the 2.34 $\pm 0.05 \mathrm{eV}$ energy of the $P_{2}$ state, and therefore are not precursors for $P_{2}$. In addition, there is a statistically significant transient intensity increase that occurs between 200-800 fs (pumped by $h \nu_{1}=3.08 \mathrm{eV}$ and probed by $h \nu_{2}=2.19 \mathrm{eV}$ ) and peaking at $250 \mathrm{fs}$ for $6 \mathrm{ML}$ and at $230 \mathrm{fs}$ for $2 \mathrm{ML}$. This delayed rise in signal at pump-probe time delays $>200$ fs can be attributed to $P_{2}$ electrons that are formed on an ultrafast timescale from $P_{1}$, of which a fraction relax to a long-lived state. The lifetime of the resulting long-lived state is coverage dependent and discussed in point (3).

(2) Energy overlap: An average of the pump-probe time-delays between 250 and 400 fs, shown in Figure 6, reveals a portion of $P_{1}$ at later pump-probe time delays overlaps with the energy region of $P_{2}$. This energy overlap between the two electronic states will facilitate electron transfer from $P_{1}$ to $P_{2}$.

(3) Wavefunction overlap: The recombination lifetime of $P_{2}$ with the metal substrate would be expected to increase as the $P_{2}$ - metal distance increases with DMSO coverage, either exponentially in a tunneling mechanism ${ }^{40}$ or with a weaker distance dependence in a multistep hopping mechanism. ${ }^{65}$ However, as seen in Figure 5(b) the lifetime of $P_{2}$ ranges from less than 0.5 $\mathrm{s}$ for $2.6 \mathrm{ML}$ to $5.2 \pm 0.9 \mathrm{~s}$ for $9.7 \mathrm{ML}$ of DMSO and saturates by approximately 6 ML to an average lifetime of 4 seconds. This saturation behavior implies that there is a competing effect that also determines the measured average lifetime of $P_{2}$ as a function of coverage. We propose this competing effect is the wavefunction overlap required for $P_{1}$ to act as a precursor state for $P_{2}$, which will decrease as a function of $P_{1}-P_{2}$ distance. While the binding sites for $P_{2}$ are likely to be found distributed over the multilayer islands, only those with wavefunction over- lap with $P_{1}$ would be populated, which effectively weights the measured average lifetime of $P_{2}$ towards binding sites closer to the 2nd monolayer and thus with shorter lifetimes. In a simple illustrative model for the two competing effects described in the supporting information, the decreasing probability of electron transfer from $P_{1}$ to $P_{2}$ as a function of distance $z$ from the 2 nd monolayer is described as a decaying exponential while the increasing lifetime of $P_{2}$ as a function of distance from the $\mathrm{Cu}$ substrate is modeled as an increasing exponential. As seen by the blue-dashed line in Figure 5(b), a least-square fit of this illustrative model to the experimental data captures the observed saturation behavior well; the blue shaded region reflects other least-square fits using the different error limits of the experimental points. Therefore the saturation in the average lifetime of $P_{2}$ as a function of coverage is consistent with a requirement for wavefunction overlap between $P_{1}$ and $P_{2}$.

We conclude that the dynamically localizing small polaron, which is strongly coupled to the metal substrate, acts as a precursor, or intermediate state, for an extremely long-lived electronic state that is weakly-coupled with the metal substrate. This long-lived state $P_{2}$ has a sufficiently long lifetime that it can undergo chemical reactions.

\section{CONCLUSION}

We observe the dynamic formation of a small polaron two monolayers from the $\mathrm{DMSO} / \mathrm{Cu}$ interface within $200 \mathrm{fs}$, similar to the periods of librational and sulfuroxygen bending modes of DMSO molecules in both solid and liquid phases. As DMSO is a common solvent in battery electrolytes, the dynamic localization observed in our experiments is highly relevant to formation of localized electrolyte electronic states near electrode surfaces. Furthermore, while the dynamically-evolving small polaron is strongly coupled to the metal surface with a lifetime of only $140 \mathrm{fs}$, and therefore has a limited efficiency for chemical reactivity, it likely acts as a precursor state for a long-lived DMSO electronic state with a lifetime of seconds. With a lifetime 13 orders of magnitude longer than that of the small polaron, this long-lived electronic state is surely capable of chemical reactivity. Our findings highlight the importance of interfacial electronic states near metal surfaces for electrochemical reactions despite their femtosecond lifetimes, as they are crucial precursors for long-lived, potentially reactive states of solvents and electrolytes.

\section{SUPPLEMENTARY INFORMATION}

Details of DMSO dosing and temperature programmed desorption used to determine DMSO coverage. An explanation of the presence of a negative-time background in the time-resolved data. Fitting of the $\mathrm{XC}$ and peak- 
shifting in the time-resolved data. Time-resolved data for 2 ML DMSO on $\mathrm{Cu}(111)$. A description of dispersion fitting. The fitting of the dispersion of $P_{1}$ for three different pump-probe time delays as well as angle-resolved images from four different pump-probe time delays at high $k_{\|}$. A description of pump-wait-probe measurements. Description of a model that captures the $P_{2}$ lifetime versus coverage trends.

\section{ACKNOWLEDGMENTS}

This work was supported through funds from the Max Planck Society. S.B.K. gratefully acknowledges support from the Alexander von Humboldt Foundation. K.B. thanks the Deutsche Forschungsgemeinschaft for postdoctoral funding. J.S. thanks Sabine Reichel for continuous fruitful discussions.

${ }^{1}$ G. Blaise, J. Electrostat. 50, 69 (2001).

${ }^{2}$ S. Stafström, Chem. Soc. Rev. 39, 2484 (2010).

${ }^{3}$ S. Fratini, D. Mayou, and S. Ciuchi, Adv. Funct. Mater. 26, 2292 (2016).

${ }^{4}$ D. Emin, Phys. Today 35, 34 (1982).

${ }^{5}$ J. L. Bredas and G. B. Street, Acc. Chem. Res. 18, 309 (1985).

${ }^{6}$ L.-C. Ku, S. A. Trugman, and J. Bonča, Phys. Rev. B 65, 174306 (2002).

${ }^{7}$ M. Zoli and A. N. Das, J. Phys. Condens. Matter 16, 3597 (2004).

${ }^{8}$ P. Szymanski, S. Garrett-Roe, and C. B. Harris, Prog. Surf. Sci. 78, 1 (2005).

${ }^{9}$ L.-C. Ku and S. A. Trugman, Phys. Rev. B 75, 014307 (2007).

${ }^{10}$ J. E. Johns, E. A. Muller, J. M. J. Frechet, and C. B. Harris, J. Am. Chem. Soc. 132, 15720 (2010).

${ }^{11}$ Z. Feng, V. Timoshevskii, A. Mauger, C. M. Julien, K. H. Bevan, and K. Zaghib, Phys. Rev. B 88, 184302 (2013).

${ }^{12}$ C. J. Chandler and F. Marsiglio, Phys. Rev. B 90, 125131 (2014).

${ }^{13}$ G. Donati, D. B. Lingerfelt, A. Petrone, N. Rega, and X. Li, J. Phys. Chem. A 120, 7255 (2016).

${ }^{14}$ T. Minato and T. Abe, Prog. Surf. Sci. 92, 240 (2017).

${ }^{15}$ K. M. Abraham, J. Electrochem. Soc. 162, A3021 (2015).

${ }^{16}$ M. A. Schroeder, N. Kumar, A. J. Pearse, C. Liu, S. B. Lee, G. W. Rubloff, K. Leung, and M. Noked, ACS Appl Mater Interfaces 7, 11402 (2015).

${ }^{17}$ N. Togasaki, T. Momma, and T. Osaka, J. Power Sources 294, $588(2015)$.

${ }^{18}$ A. V. Sergeev, A. V. Chertovich, D. M. Itkis, A. Sen, A. Gross, and A. R. Khokhlov, J. Phys. Chem. C 121, 14463 (2017).

${ }^{19}$ D. Martin, A. Weise, and H.-J. Niclas, Angew. Chemie Int. Ed. English 6, 318 (1967).

${ }^{20}$ W. S. MacGregor, Ann. NY Acad. Sci. 141, 3 (1967).

${ }^{21}$ A. Shen and J. E. Pemberton, J. Electroanal. Chem. 479, 21 (1999).

${ }^{22}$ A. Shen and J. E. Pemberton, J. Electroanal. Chem. 479, 32 (1999).

${ }^{23}$ B. Sexton, N. Avery, and T. Turney, Surf. Sci. 124, 162 (1983).

${ }^{24}$ S. K. Si and A. A. Gewirth, J. Phys. Chem. B 104, 10775 (2000).

${ }^{25}$ N. Ikemiya and A. A. Gewirth, J. Phys. Chem. B 104, 873 (2000).

${ }^{26}$ M. L. Strader, S. Garrett-Roe, P. Szymanski, S. T. Shipman, J. E. Johns, A. Yang, E. Muller, and C. B. Harris, J. Phys. Chem. C 112, 6880 (2008).

${ }^{27}$ N.-H. Ge, C. M. Wong, R. L. Lingle Jr., J. D. McNeill, K. J. Gaffney, and C. B. Harris, Science 279, 202 (1998).

${ }^{28}$ J. Stähler, C. Gahl, U. Bovensiepen, and M. Wolf, J. Phys. Chem. B 110, 9637 (2006).

${ }^{29}$ M. Muntwiler, Q. Yang, and X.-Y. Zhu, J. Electron Spectros. Relat. Phenomena 174, 116 (2009).
${ }^{30}$ C. H. Schwalb, M. Marks, S. Sachs, A. Schöll, F. Reinert, E. Umbach, and U. Höfer, Eur. Phys. J. B 75, 23 (2010).

${ }^{31}$ J. Stähler, M. Meyer, U. Bovensiepen, and M. Wolf, Chem. Sci. 2, 907 (2011).

${ }^{32}$ A. Migani, D. J. Mowbray, A. Iacomino, J. Zhao, H. Petek, and A. Rubio, J. Am. Chem. Soc. 135, 11429 (2013).

${ }^{33}$ J. W. Kim, H. Park, and X. Zhu, J. Phys. Chem. C 118, 2987 (2014).

${ }^{34}$ J. Stähler, J.-C. Deinert, D. Wegkamp, S. Hagen, and M. Wolf, J. Am. Chem. Soc. 137, 3520 (2015).

${ }^{35}$ T. Wang, C. Caraiani, G. W. Burg, and W.-L. Chan, Phys. Rev. B 91, 041201 (2015).

${ }^{36}$ D. Emin and T. Holstein, Phys. Rev. Lett. 36, 323 (1976).

${ }^{37}$ J. T. Devreese, in Digit. Encycl. Appl. Phys. (WILEY-VCH Verlag GmbH \& Co KGaA, Weinheim, Germany, 2003) pp. 383-412.

${ }^{38}$ D.-F. Feng and L. Kevan, Chem. Rev. 80, 1 (1980).

${ }^{39}$ A. Kumar, J. A. Walker, D. M. Bartels, and M. D. Sevilla, J. Phys. Chem. A 119, 9148 (2015).

${ }^{40}$ J. Stähler, M. Meyer, D. O. Kusmierek, U. Bovensiepen, and M. Wolf, J. Am. Chem. Soc. 130, 8797 (2008).

${ }^{41}$ A. J. Shearer, D. E. Suich, B. W. Caplins, and C. B. Harris, J. Phys. Chem. C 119, 24417 (2015).

${ }^{42}$ U. Bovensiepen, C. Gahl, J. Stähler, M. Bockstedte, M. Meyer, F. Baletto, S. Scandolo, X. Y. Zhu, A. Rubio, and M. Wolf, J. Phys. Chem. C 113, 979 (2009).

${ }^{43}$ M. Bertin, M. Meyer, J. Stähler, C. Gahl, M. Wolf, and U. Bovensiepen, Farad. Discuss. 141, 293 (2009).

${ }^{44}$ J. Stähler, C. Gahl, and M. Wolf, Acc. Chem. Res. 45, 131 (2012).

${ }^{45}$ S. B. King, D. Wegkamp, C. Richter, M. Wolf, and J. Stähler, J. Phys. Chem. C 121, 7379 (2017).

${ }^{46}$ U. Bovensiepen, H. Petek, and M. Wolf, eds., Dynamics at solid state surfaces and interfaces, Vol. 2 (Wiley-VCH, 2012) pp. 155180.

${ }^{47}$ D. Wegkamp, Ultrafast Electron Dynamics and the Role of Screening, Ph.D. thesis, Freie Universität Berlin (2014).

${ }^{48}$ W. Berthold, F. Rebentrost, P. Feulner, and U. Höfer, Applied Physics A 78, 131 (2004).

${ }^{49}$ H. Petek, H. Nagano, M. Weida, and S. Ogawa, Chem. Phys. 251, 71 (2000).

${ }^{50}$ J. Güdde, W. Berthold, and U. Höfer, Chem. Rev. 106, 4261 (2006).

${ }^{51}$ T. Kumagai, Personal Communication (2017).

${ }^{52}$ C. Schröter, B. Roelfs, and T. Solomun, Surf. Sci. 380, L441 (1997).

${ }^{53}$ S. A. Kirillov, M. I. Gorobets, M. M. Gafurov, M. B. Ataev, and K. Rabadanov, J Phys Chem B 117, 9439 (2013).

${ }^{54}$ U. Bovensiepen, C. Gahl, and M. Wolf, J. Phys. Chem. B 107, 8706 (2003).

${ }^{55}$ J. Patterson and B. Bailey, Solid-State Physics: Introduction to the Theory, 2nd ed. (Springer-Verlag, Heidelberg, Germany, 2010).

${ }^{56}$ X.-Y. Zhu, J. Phys. Chem. B 108, 8778 (2004).

${ }^{57}$ J. Savolainen, F. Uhlig, S. Ahmed, P. Hamm, and P. Jungwirth, Nat. Chem. 6, 697 (2014).

${ }^{58}$ A. Kovalenko, Cond. Mat. Phys. 18, 32601 (2015).

${ }^{59}$ K. L. Sebastian, A. Chakraborty, and M. Tachiya, J. Chem. Phys. 119, 10350 (2003).

${ }^{60}$ J. R. Durig, C. M. Player, and J. Bragin, J. Chem. Phys. 52, 4224 (1970).

${ }^{61}$ P. P. Wiewiór, H. Shirota, and E. W. Castner, J. Chem. Phys. 116, 4643 (2002).

${ }^{62}$ M. L. Strader and S. E. Feller, J. Phys. Chem. A 106, 1074 (2002).

${ }^{63}$ D. Sanchez-Portal, J. Stähler, and X. Zhu, in Dynamics at solid state surfaces and interfaces, Vol. 2, edited by U. Bovensiepen, H. Petek, and M. Wolf (Wiley-VCH, 2012) Chap. 4, pp. 155-180.

${ }^{64} \mathrm{We}$ observe a linear real-time decrease in the intensity of peak $P_{2}$ with photo-illumination. Neither the ultrafast dynamics nor the pump-wait-probe measurements of $P_{2}$ are effected by the 
intensity changes. Any real-time effects to the intensity of peak $P_{2}$ were mitigated in data collection by looping through pumpprobe time delays in both the forward and reverse directions followed by summing both delay directions. The origin of this intensity decrease is currently under investigation.

${ }^{65}$ B. Giese, Acc. Chem. Res. 33, 631 (2000). 